\title{
Creep Bending Analysis of Combined Rotor for Gas Turbine Considering Contact Stiffness
}

\author{
Haibiao Zhang ${ }^{1, a}$ Ailun Wang ${ }^{1, b}$ \\ ${ }^{1}$ State Key Laboratory of High Performance Complex Manufacturing, Central South University, \\ Changsha 410083, China \\ azhbcsu@163.com, ${ }^{\mathrm{b}}$ walalz@sohu.com
}

\section{Keywords:combined rotor;creep Bending;gas turbine;high temperature;ansys}

\begin{abstract}
Combined rotor is the core part of gas turbine and works in high temperature and high rotating speed environment in a long-term,creep bending of the combined rotor will make great effects on the gas turbine's performance. To study the creep bending properties of gas turbine's combined rotor considering the contact stiffness, in this paper, virtual material layer was used to equal the stiffness of contact interface when building the finite element model of combined rotor, and choosed the Norton creep constitutive model to carry out heat-solid coupling analysis with the FEA software ansys under a given temperature and stress fields. Firstly, the creep effect of balanced combined rotor of a 300MW gas turbine was calculated, got the main creep area of combined rotor and the creep quantitative data changing with time. Then, the relationship between creep bending amount of combined rotor with eccentric mass and time was researched.Results show that, the creep mainly occurred on shaft between twelfth wheel of the compressor and third wheel of the turbine; when the combined rotor got a eccentric mass, it would bend, and with time went on, the amount of eccentricity increased gradually, the bending rate also increased, and the bending rate of the 17 th wheel of the compressor was the highest. The results of this dissertation will support reference for structure design and vibration analysis of the combined rotor.
\end{abstract}

\section{Introduction}

More than $80 \%$ of faults of Rotating Machinery is caused by Rotor Vibration. ${ }^{[1]}$ Vibration of the combined rotor is one of the main cause of the faults of Gas Turbine, since vibration of the Gas Turbine has close relationship with the safety of the shaft system which is the most exorbitant part of Gas Engine. Therefore, the vibration of Gas Turbine has always been payed highly attention to by Gas Turbine Power Plant. ${ }^{[2]}$ The unbalance of rotor is the main excitation source of Rotating Machinery. Bending of rotor or deviation between rotors' center is one of causes which lead to vibration of gas turbine or other Rotating Machinery, and it makes the shafting can not be a smooth continuous line. Bending of rotor is not only one of the most difficult to eliminate but also one of the most important fault sources of vibration. ${ }^{[3]}$ As one of the main parts of Gas Turbine, once the combined rotor occurs large shafting bend, ${ }^{[4]}$ the shafting will vibrate heavily and do harm to the safety and stability of Gas Turbine.

The causes of rotor bending mainly includes material, design, transportation, operation, etc. ${ }^{[5]}$ At present, a large amount of studies on rotor bending are for Gas Turbine, reference ${ }^{[6]}$ analysed asymptotic bending of high-medium pressure rotor, the bending mechanism of combined rotor is more complicated due to the complexity of its structure and stiffness of contact interface.

Combined rotor of Gas Turbine is always working in high temperature environment, therefore, the creep characteristics of material has vital effect on combined rotor. In the engineering, creep characteristics of material can lead to non-normal operation of the units. For example, in June 1964, Tennessee of America, an accident happened because of creep characteristics of combined rotor. 
The investigation showed that the radial displacement between blade and wheel disc exceed the clearance between blade and casing. ${ }^{[7]}$ In our country, there is also some little study on creep characteristics of combined rotor at present. Wenrui $\mathrm{Wu}^{[8]}$ etc analyzed the creep characteristics of high-medium pressure rotor in high temperature environment. Yanping Sun ${ }^{[9]}$ etc analyzed the creeping bending of intermediate pressure rotor, in theory, they proved that high temperature creep leads to rotor bending. According to combined rotor of a F Gas Turbine, considering centrifugal force, extra force caused by uneven material, temperature load, torque, eccentric force of rotating shaft, etc. ${ }^{[10]}$ Creep bending changes with time can be got by studying creep characteristics and bending mechanism.

\section{Finite element model of combined rotor}

Combined rotor has different numbers of tied rod and wheel disc according to model number. The study object of this paper is gas turbine rotors designed by Dong Fang Turbine Company Limited. Figure 1 shows the model of circumferential rod fastening rotor (combined rotor). Combined rotor is composed of compressor, combustion chamber and turbine. Compressor consists of 17 wheel discs and 12 tied rods. Turbine consists of 4 wheel discs and 12 tied rods. Combustion chamber is between the compressor and turbine. The wheel discs on compressor and turbine are connected with 12 tied rods on which the pre-load is applied, many contact interfaces exist between wheel discs. The material density of combined rotor is $7850 \mathrm{~kg} \bullet \mathrm{m}^{-3}$, the elastic modulus of wheel disc and rotating shaft is $210 \mathrm{GPa} .47852$ units and 189952 nodes can be got after meshing the combined rotor. Physical parameters of combined rotor are showed in table 1.

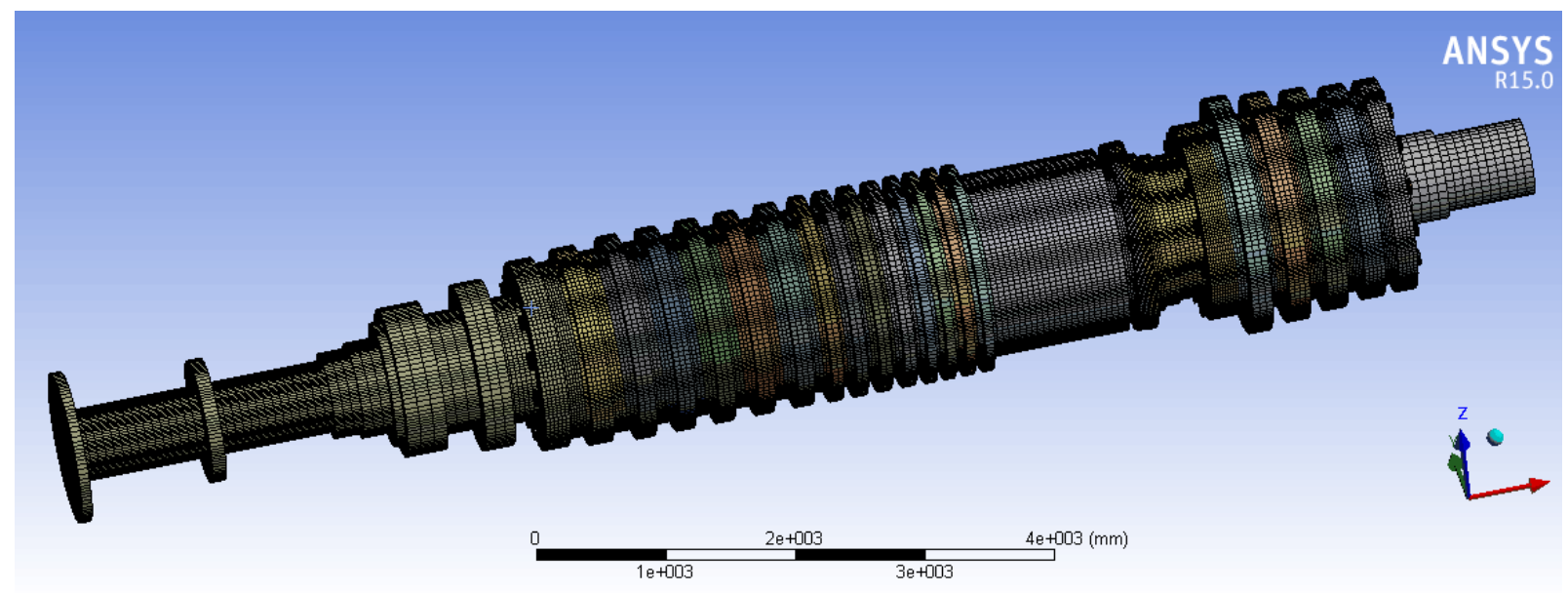

Fig.1 The finite element model of the combined rotor 
Table 1 Physical parameters of the combined rotor

Physical parameters

Value $(\mathrm{mm})$

Total length of the rotor: $l_{\text {rotor }}$

11582

The thickness of 4-7 wheel of compressor : $l_{\text {disk4-7 }}$

The thickness of 8-11 wheel of compressor: $l_{\text {disk8-11 }}$

The thickness of 12-14 wheel of compressor : $l_{\text {disk12-14 }}$

The thickness of 15-17 wheel of compressor: $l_{\text {disk15-17 }}$

Length of Combustor: $l_{\text {combustor }}$ 1905

The thickness of 1-2 wheel of turbine: $l_{\text {disk } 1-2}$ 304

The thickness of 3-4 wheel of turbine: $l_{\text {disk3-4 }}$

Wheel diameter: $d_{\text {disk }}$

Rod diameter : $d_{\text {rod }}$ 80

Rod length: $l_{\text {rod }}$ 3740

The existence of contact interface of the wheel is the biggest difference between the combined rotor and the integral rotor. It leads to partial stiffness change of the combined structure, different interface status directly affect the vibration characteristics of the structure ${ }^{[11]}$.

To do dynamic analysis of combined institution accurately, the contact stiffness of rough surface needs to be obtained. In this paper, cross-scale calculation method of combination of micro unit model with the same interface roughness analysis and macroscopic size was used, as reference ${ }^{[12]}$ proposed.

Using the formula (1) (2) to obtain the interface normal contact stiffness at preload of the combined rotor.

$$
\begin{aligned}
& K=\frac{S_{1}}{S_{2}} k_{\mathrm{n}} \\
& F=P \times S_{1}
\end{aligned}
$$

Where in, $S_{1}$-- contact interface area;

P-- the interface pressure at preload;

$\mathrm{k}_{n--}$ normal contact stiffness of micro unit model;

F-- preload of combined rotor;

$\mathrm{K}-$ - interface normal contact stiffness.

In this paper, a certain amount of equivalent treatment of the contact interface was done when doing dynamics analysis of the combined rotor. Binding surface under external loads, when the normal stiffness is known, according to the strain energy equivalence principle, as shown in Figure

2 , the contact stiffness equivalent to a virtual material layers, elastic modulus $\bar{E}$,the equivalent formula is:

$$
\bar{E}=K h / S_{1}
$$


Where, $\mathrm{h}$ is the thickness of the virtual material layer; $\mathrm{K}$ is the stiffness of the contact interface; $S_{1}$ is the area of the contact interface.

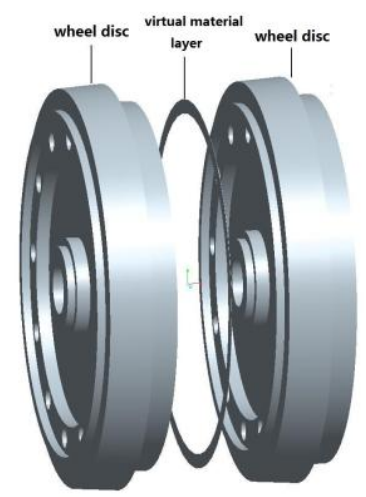

Fig.2 Contact interface equivalent to a virtual material layer

Since in gas turbine combined rotor, only the contact between compressor disks is surface-to-surface, which transfers torque by friction, while the turbine wheel is connected by the face tooth. Considering the model is more realistic rotor rod, the contact interface of the turbine section and the rod through the hole is bonded, considering the contact interface of the compressor only.

Table. 2 shows the calculation results of the model and experimental results of heavy gas turbine. Results prove that there is no significant difference between the model and the actual model, so the model is reliable.

Table 2.Comparisons of combined rotor model's calculation results and experimental results calculation results $(\mathrm{Hz})$ experimental results $(\mathrm{Hz})$

$\begin{array}{ccc}\text { first-order bending } & 20.8 & 17.5 \\ \text { second-order bending } & 43.1 & 41.7 \\ \text { third-order bending } & 57.5 & 55.8\end{array}$

\section{Creep model and parameters}

Generally, creep is divided into three stages: the initial stage, the stable stage and the acceleration stage. Since the creep of gas turbine combined rotor usually lasts for a long time, and most of the time it stays in the stable stage, so the Norton creep model was adopted in this paper(the second stage of creep), its constitutive equation as formula (4):

$$
\dot{\varepsilon_{\mathrm{c}}}=C_{1} \sigma^{C_{2}} e^{\left(-C_{3} / T\right)}
$$

Where $\dot{\varepsilon_{\mathrm{cr}}}, \sigma, T$ is strain derivation, loading stress and thermodynamic temperature respectively, and $C_{1}, C_{2}, C_{3}$ are parameters of the creep formula.

According to the engineering application,in this article choosed GH4169, as material of combined rotor, and according to the creep experiments in, the three parameters in formula (4) was obtained through least square method. Parameters of the norton model are as follows: 
Table 3 Parameters of creep equation

\begin{tabular}{cccc}
\hline Parameters & $C_{1}$ & $C_{2}$ & $C_{3}$ \\
\hline Value & $2.14 \times 10^{-9}$ & 10.17 & 50825 \\
& & & \\
\hline
\end{tabular}

\section{Calculation of creep deformation}

Due to the long-term working in high temperature of the combined rotor, the combined rotor disks will creep because of its centrifugal force. The creep calculation of combined rator are devided into two parts in this section, firstly study the creep deformation of balanced combined rotor and then study the creep bending of combined rotor with a eccentric mass.

\subsection{Creep analysis of balanced combined rotor}

When doing thermal analysis of the balanced rotor, no attention was payed to the axial temperature difference between Single-stage Roulette in this paper. The operation temperature distribution of combined rotor is shown in Figure 3, and Figure 4 shows the location of creep data collection.

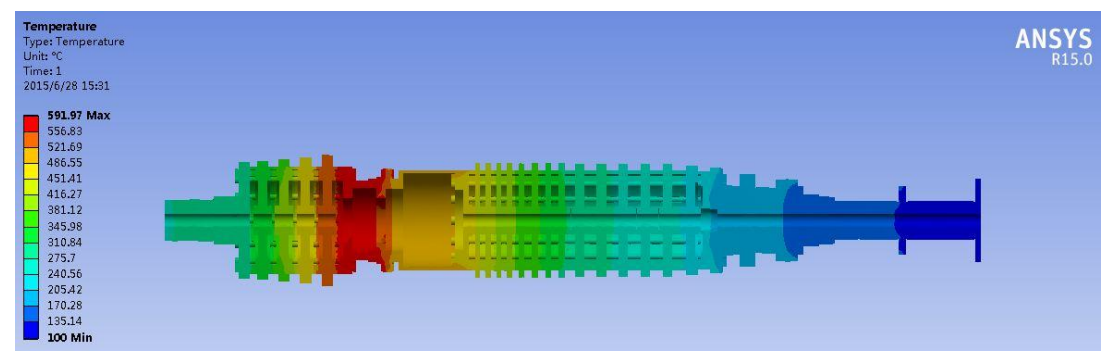

Fig.3 Operation temperature distribution of combined rotor

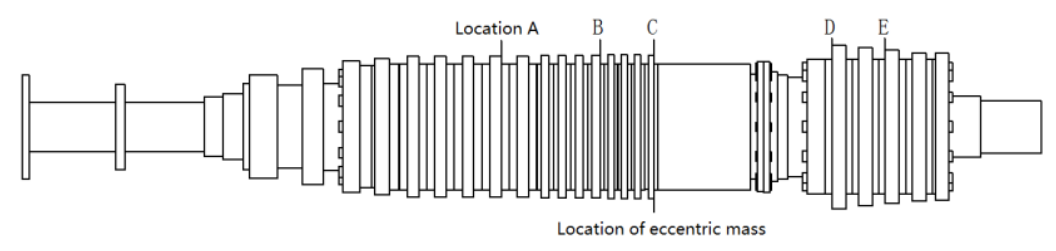

Fig.4 Location of combined rotor creep data collection

Take the operating speed of 3000rpm of combined rotor for example, the stress distribution is shown in Fig 5, Fig 6 shows the creep strain with combined rotor working for 10000h, and Fig 7 is a creep elongation curve of each collection point.

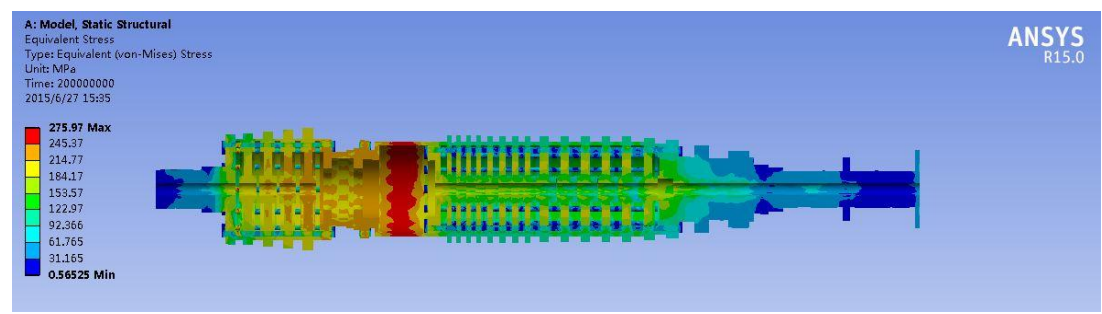

Fig.5 Stress distribution of combined rotor 


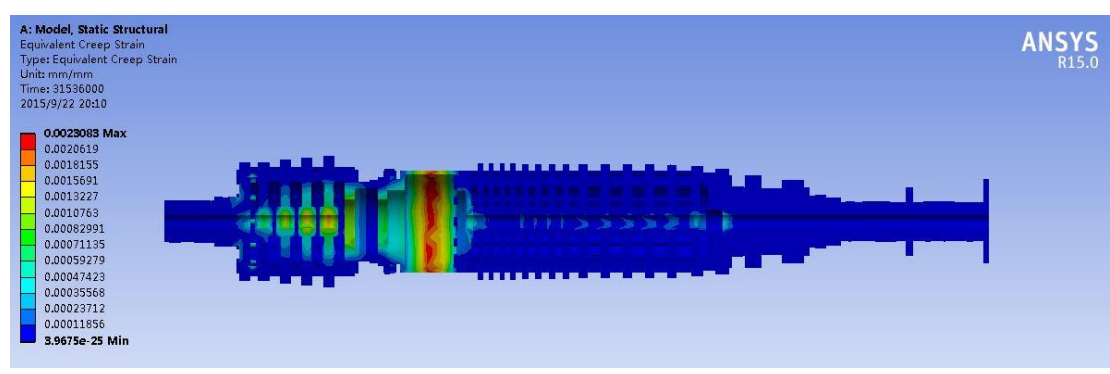

Fig.6 creep strain diagram of combined rotor

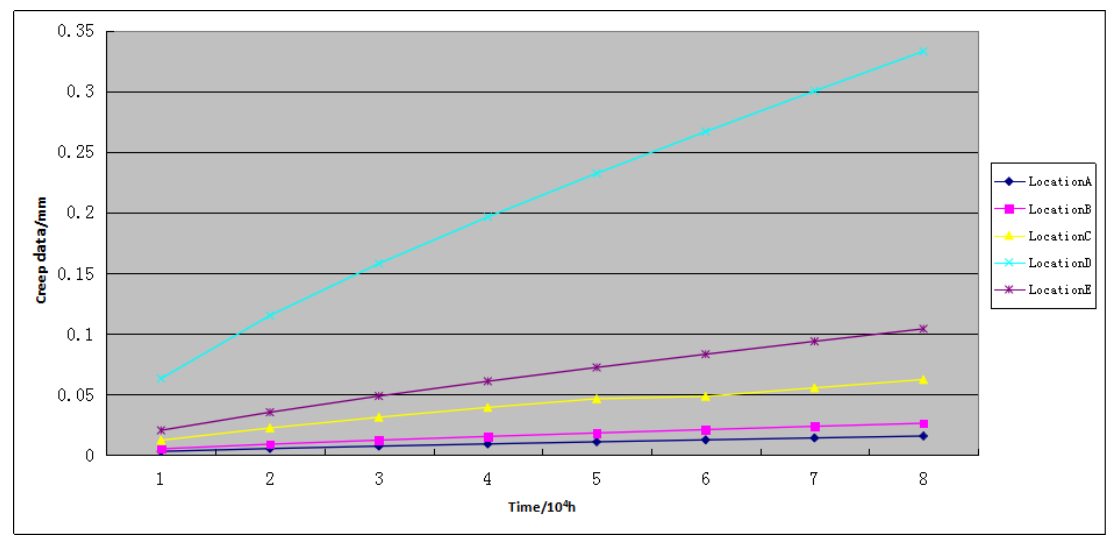

Fig. 7 creep elongation curve of each collection point

It can be seen from Fig 6 and 7:

1.The composite rotor creep occurs mainly on the compressor side between 12 th to the turbine side 3rd grade;

2.With time goes by, creep rate of combined rotor decreases.

3.In the first level on turbine side, the creep rate is the fastest.

\subsection{Combination of eccentric rotor bending creep analysis}

With the gradual development of creep bending, bending quantity distribution of the combined rotor changes, leading to its eccentric load change. Therefore, taking into account changes of bending, updated the eccentric load each time step, obtained the bending trend of high pressure rotor by iteration.

\subsubsection{Mass unbalance causes of combined rotor analysis}

Combined rotor's mass unbalance is caused by many reasons, structure, system design, installation, and mainly including several aspects as follows:

1.Mass eccentricity still exist after dynamic unbalance of combined rotor;

2.Effect of residual stresses. Due to reasons such as material, heat treatment, residual stresses inside the rotor disappear when operating, which once reaches to a serious stage can cause permanent bending of the shaft;

3.Due to unfit transportation or storage, permanent bending will be caused by mechanical forces;

4.Long-time running causes severe circumferential imbalance worn or fall off to combined rotor's blades;

5.Long-time running causes different levels of fouling to rotor center and rotor blade's surface.

Mainly based on the allowing unbalance formula of rotor after dynamic balance, initially got the combined rotor' $s$ unbalance mass and did analysis of creep bending, eccentric mass in this paper is shown in Fig 4 , set on the 17th stage of the compressor side wheel.

\subsubsection{Bending value calculation method}

Supposing measuring bending value on circle COD as shown in fig 8 , the displacement 


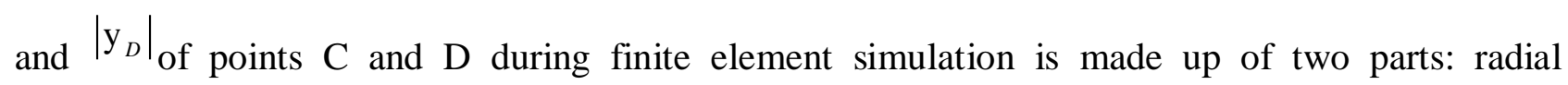
displacement caused by the centrifugal force and bent displacement caused by the creep. When the rotor is static, the locations of points $\mathrm{C}$ and $\mathrm{D}$ are as shown in Fig 8. When the rotor is rotating, due to the effect of centrifugal force, points $\mathrm{C}$ and D moves to $C_{1}$ and $D_{1}$ respectively. In the presence of long time creep bending, rotor will bend and points $C_{1}$ and $D_{1}$ will move to $C_{2}$ and $D_{2}$ respectively.

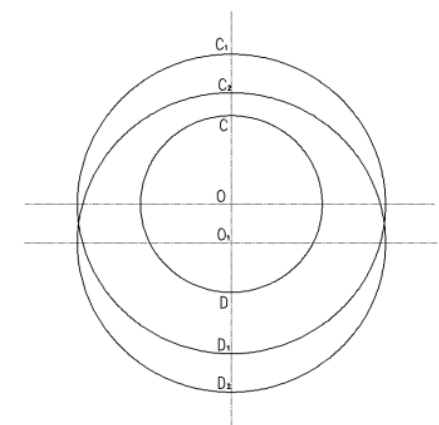

Fig.8 Creep bending forming diagram

Define the offset of the center point of the rotor $\mathrm{O}$ as the bending of rotor, the bending calculation method of the rotor is as formula 6 .

$$
D_{\text {shake }}=\frac{\left|y_{D}\right|-\left|y_{C}\right|}{2}
$$

\subsubsection{Calculation of creep bending}

Given the mass eccentricity of the combined rotor, do creep bending analysis of the combined rotor. Read the bend amount of 5 sections as A, B, C, D and E in Fig 9:

Fig 9 shows that:

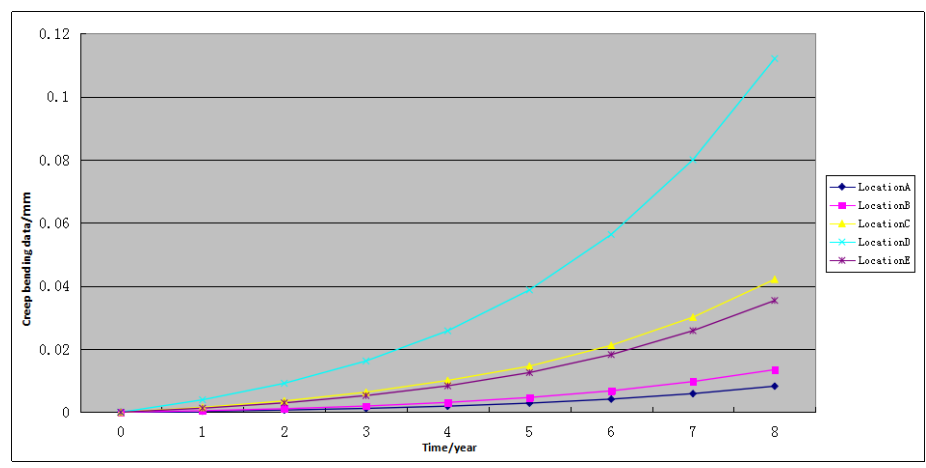

Fig.9 creep bending data of each collection point

1.With time goes by, creep bending rate of all parts of combined rotor with eccentric mass show a trend of increase;

2.Creep bending rate of the first level on turbine side is the largest. And the creep bending almost reaches the allowing value in engineering at the time of $80000 \mathrm{~h}$.

\section{Conclusion}

Vibration of combined rotor is always the focus and emphasis and bending is one of the main reasons that cause vibration of the combined rotor, so the study of the mechanism of the creep 
bending of combined rotor is of high value for protecting gas turbine' $\mathrm{s}$ high efficiency and safe operation. Modeling and simulation research was used in this article, to study the creep mechanism of a balanced combined rotor and a combined rotor with mass eccentricity.

Results show that:

1.The creep of combined rotor occurs mainly between the 12th of compressor side and the 3rd grade of turbine side;

2.The creep rate of the first level on turbine side is the fastest, which reaches the maximum value $0.333 \mathrm{~mm}$ in the seventh year;

3.The creep bending rate of combined rotor with eccentric mass increases with time;

4.The creep bending rate of the 17 th level of compressor side is the largest. And the creeping bent reaches the maximum allowing value in engineering. Enough attention should be payed to.

\section{Acknowledgments}

This research is supported by the National Basic Research Program of China (973 program: 2013CB035706).

\section{References}

[1] Yuezhen Chen.Vibration monitoring and analysis of rotating machinery[J].Gasturbine technology,2010,3(1):40-44

[2] Mauro Venturini, Nicola Puggina. Prediction reliability of a statistical methodology for gas turbine prognostics[J]. Journal of Engineering for Gas Turbines and Power, 2012, 134(10).

[3] Jinnan Liu,Lidong He.Research on Bending rotor vibration faults technology eliminates single plane Active Balancing[J].Thermal power engineering, 2006,21(2):166-169

[4] Jianguo Dong,Jianbo Tian.Vibration analysis of turbine[J].Gas Turbine Technology, 2004,17 (2) : 63-66

[5] Yahui Cui,Junjie Zhang,Dongpeng Jiang.Research on creep characteristic of a supercritical 600 MW unit turbine rotor[J].Thermal power generation, 2013,42(12):69-71

[6] Junjie Zhang,Yahui Cui.Gradual bend occurred on HP-IP rotor in 600MW level unit:fault diagnosis and treatment[J].Thermal power generation,2013,42(7):105-108

[7] Xiaying Mu.Creep Mechanics[M].Xi'an Jiaotong university Press.1990.6

[8] Wenrui Wu,Weizhe Wang,Puning Jiang,Yingzheng Liu.Strength Analysis of High-temperature Creep for a 660MW Ultra-Supercritical Steam Turbine High-pressure Rotor [J].Journal of Power Engineering, 2009,29(2).99-103

[9] Yanping Sun,Chunfeng Pang.Research on Rotor Creep Properties under Bending Residual Stress [J].Journal of mechanical engineering,2014,50(10): 162-165

[10] Goerke D, Willner K. Normal contact of fractal surfaces-experimental and numerical investigations[J]. Wear, 2008, 264(7):589-598.

[11] Huiguang Li,Heng Liu,Lie Yu.Contact Stiffness of Rough Mechanical Joint Surface.Journal of Xi'An Jiaotong university.2011,45(6):69-74.

[12] Mccool J I. The distribution of microcontact area, load, pressure and flash temperature under Greenwood-Williamsom model[J]. ASME Journal of Tribology, 1988, 110(1):106-111. 